\title{
In-vitro Susceptibility of Mycoplasma capricolum Subsp. capripneumoniae Pakistan Strain to Commercially Available Quinolones
}

\author{
Mumtazur Rahman ${ }^{1}$, Farhan Anwar Khan ${ }^{1, *}$, Umar Sadique ${ }^{1}$, Ijaz Ahmad ${ }^{1}$, \\ Shakoor Ahmad ${ }^{1}$, Faisal Ahmad, ${ }^{1,2}$, Hayatullah Khan ${ }^{1,3}$, Muhammad Saeed ${ }^{1}$, \\ Faiz Ur Rehman', Ibrar Hussain ${ }^{1}$, M. Faraz Khan', M. Izhar ul Haque ${ }^{1}$ and \\ Hanif-ur-Rehman ${ }^{1,3}$ \\ ${ }^{1}$ College of Veterinary Sciences, Department of Animal Health, Faculty of Animal \\ Husbandry and Veterinary Sciences, 25120, The University of Agriculture, \\ Peshawar, Khyber Pakhtunkhwa, \\ ${ }^{2}$ Directorate of Livestock and Dairy Development (Extension), Peshawar, 25000, \\ Khyber Pakhtunkhwa \\ ${ }^{3}$ Directorate of Livestock and Dairy Development (Research), Peshawar, 25000, \\ Khyber Pakhtunkhwa
}

\begin{abstract}
A B S T RA C T
Contagious caprine pleuropneumonia (CCPP) caused by Mycoplasma capricolum subsp. capripneumoniae (Mccp) is a highly contagious and deadly disease of small ruminants, especially goats. It is an economically important disease, causing high morbidity and mortality in goats. More recently Mccp Pakistan strain has been identified and isolated by our laboratory. Alarmingly, antimicrobials in animals are inappropriately using and resultantly leading to antimicrobial resistance (AMR) including mycoplasmas, which are causing serious infections in ruminants. Since the growth curve of our local strain and its susceptibility to commonly using antimicrobials is unknown. Therefore, this study was aimed to evaluate the growth curve and susceptibility of Mccp against commercially available quinolones in Pakistan by broth microdilution method. Quinolones including moxifloxacin, levofloxacin, ciprofloxacin, and enrofloxacin were selected for in vitro susceptibility of Mccp Pakistan strain. The concentration for each drug was measured distantly depending on high concentration available in the market and was then used in various concentrations ranges from higher to lower i.e. moxifloxacin $80-0.156 \mu \mathrm{g} / \mathrm{ml}$, levofloxacin $250-0.488 \mu \mathrm{g} / \mathrm{ml}$, ciprofloxacin and enrofloxacin $50-0.097 \mu \mathrm{g} / \mathrm{ml}$. The resultant effective antimicrobial by minimum inhibitory concentration (MIC) and minimum mycoplasmacidal concentration (MMC) value was ciprofloxacin having lowest MIC and MMC value $(0.390 \mu \mathrm{g} / \mathrm{ml}$ and $0.781 \mu \mathrm{g} / \mathrm{ml})$, respectively. It was concluded that ciprofloxacin had best mycoplasmacidal and mycoplasmastatic activities among tested chemotherapeutics agents against Mccp. Ciprofloxacin could be more effective antimicrobial in field against Mccp infection in goat.
\end{abstract}

Article Information
Received 31 December 2019
Revised 11 February 2020
Accepted 04 March 2020
Available online 07 January 2021
Authors' Contribution
MR, FAK, and US designed and
conceived the study. MR, IB, HK, FA,
and MFK carried out the research.
MR, FAK, IA, SA, and MIH analyzed
the data. MR, FAK, MS, and FR wrote
the manuscript. FAK, US, and HR
critically reviewed and revised the
manuscript.
Key words
Antimicrobials, CCPP, Goats, Mccp,
PCR, Quinolones.

\section{INTRODUCTION}

$\mathrm{C}$ Contagious Caprine Pleuropneumonia (CCPP) is an economically important disease of goats. CCPP is in OIE list of notifiable terrestrial and aquatic animal diseases, causing a considerable economic loss in terms of high morbidity and mortality in goat flocks in underdeveloped countries including Pakistan. Goat farming carries enormous significance in the rural economy, especially for non-agricultural poor needy people (Rehman et al., 2017). This category of animal is exposed to various problems in the country including petty management, underfeeding, communicable and non-communicable

\footnotetext{
* Corresponding author: farhan82@aup.edu.pk 0030-9923/2021/0002-0409\$9.00/0

Copyright 2021 Zoological Society of Pakistan
}

diseases. In a group of communicable diseases, CCPP is a trans-boundary disease and is responsible for up to $60-80 \%$ mortality in an exposed group of caprine (OIE, 2008). CCPP is caused by a member of minimal, simplest and slow-growing class mollicutes known as Mycoplasma capricolum subsp. capripneumoniae (Mccp), further classified as a member of the Mycoplasma mycoides cluster (Swai et al., 2013). Clinical signs of the disease comprised low to very harsh cough, mucopurulent nasal discharge, dullness, debilitation, loss of appetite and elevated temperature. Nonavailability of an effective vaccine makes control of the disease difficult. However, chemotherapy is a major approach in the control strategy of disease (Gomez-Martin et al., 2013). Therefore the selection of antimicrobial for reliable and efficient chemotherapy, control and prevention of disease caused by mycoplasma is mandatory. As these bacteria lack cell 
walls, therefore they are resistant to some antibiotics, such as penicillin's and cephalosporins, that inhibits cell wall synthesis of bacteria. Treatment of the respiratory disease is mostly carried out by commercially available antibiotic agents with fluctuating success. Due to the mixed and inappropriate doses of antimicrobials, infection most probably leads to development of resistance in mycoplasma, which ultimately leads to therapeutics failure (Scott and Menzies, 2011; Laura et al., 2006). Another major reason of antibiotic failure and progression of bacterial resistance in developing countries are quacks, inferior quality of drugs, inappropriate treatment duration and quick substitution of therapeutic agents (Canton et al., 2013; Mathew et al., 2007). The indiscriminate and irrational use of common antimicrobials in animals may develop wide range of resistance against the pathogenic bacteria. Mycoplasma species are resistant to most antimicrobial drugs because of the lack of cell wall. The antimicrobial agents which act on nucleic acid and protein synthesis are commonly used against Mycoplasmosis in small ruminants (Clothier et al., 2012). The most commonly used antibiotics are enrofloxacin, tylosin, gentamycin, and oxytetracycline with different degree of success (Laura et al., 2006). The continuous use of antibiotics in small ruminants irrespective of proper diagnosis leads to an increased antibiotics resistance (Scott and Menzies, 2011). Different pathogenic Mycoplasma species develop resistance against spectinomycin, tylosin and oxytetracycline in many countries of Europe (Ayling et al., 2000). In Pakistan, many chemotherapeutics agents are used in respiratory infection of small ruminant without any veterinary supervision, so the potential for the incidence of resistance to antibiotics should be considered. The goal of this research was to analyzed in vitro susceptibility of frequently using members of quinolone group against Mccp local strain. Local strain of Mccp causing CCPP was isolated recently by our laboratory for the first time in Pakistan. Outcomes of this research will help veterinarians and poor farmers in proper treatment of Mccp infection.

\section{MATERIALS AND METHODS}

Cultivation of Mycoplasma capricolum subsp. capripneumoniae local strain

The study was carried out in Pathology Laboratory of Department of Animal Health, Faculty of Animal Husbandry and Veterinary Sciences, The University of Agriculture Peshawar. The sampling was carried out in the Northern and Central zones of Khyber Pakhtunkhwa, Pakistan. The Mccp local strain was isolated from lungs, pleural fluid and tracheal samples of infected goats with CCPP. The local strain isolated by our laboratory was used in this study (unpublished data). The Mccp local strain was cultivated in PPLO (2.1\%) (Difco), supplemented with $20 \%$ de-complemented horse serum (Thermo-scientific), $10 \%$ fresh yeast extract (Difco), $0.2 \%$ glucose (Difco), $0.4 \%$ sodium pyruvate (Sigma Aldrich), 80000 IU/100 $\mathrm{ml}$ Penicillin G (Sigma Aldrich) under $5 \% \mathrm{CO}_{2}$ at $37^{\circ} \mathrm{C}$ conditions for 7-10 days and examined periodically for color change, turbidity and whirling movement of Mccp growth. The slight turbid or color changed sample was subcultured on PPLO agar for the appearance of fried egg colonies of 0.1 to $0.3 \mathrm{~mm}$ in diameter having dense elevated center was suggestive of Mccp species colonies (OIE, 2013).

Confirmation of Mycoplasma capricolum subsp. capripneumoniae Pakistan strain by PCR

The DNA from mccp culture was extracted by boiling method as described elsewhere (Tabatabaei et al., 2014). The harvested Mccp culture was centriguged at $15000 \mathrm{x} \mathrm{g}$ for $5 \mathrm{~min}$ and the supernatant was discarded. The pellet was resuspended in distilled water and boiled for $5 \mathrm{~min}$. The sample was again centrifuge at 15000 $\mathrm{x} \mathrm{g}$ for $1 \mathrm{~min}$. The supernatant was used as a DNA template. The species specific primers, Mccp-Spe-F: 5' - ATCATTTTTAATCCCTTCAAG-3' and Mccp-speR:5' - TACTATGAGTAAGAGTAATTATATTAATATATGCAA- 3' were used for the molecular conformation of Mccp by PCR as described previously (Woubit et al., 2004; Shah et al., 2017). A $25 \mu$ l PCR reaction was prepared by mixing $10 \mu \mathrm{l}$ of master mix, $1.75 \mu \mathrm{l}$ of each primer, $3.5 \mu \mathrm{l}$ of DNA template and $8 \mu \mathrm{l}$ of PCR water. The PCR conditions was set at an initial denaturation step of $3 \mathrm{~min}$ at $95^{\circ} \mathrm{C}$, followed by 34 cycles of $30 \mathrm{sec}$ at $95^{\circ} \mathrm{C}$, $15 \mathrm{sec}$ at $53^{\circ} \mathrm{C}$ and $15 \mathrm{sec}$ at $72^{\circ} \mathrm{C}$. The final extension step was for $5 \mathrm{~min}$ at $72^{\circ} \mathrm{C}$. The PCR product was then run on gel electrophoresis using $1 \%$ molecular grade agarose gel with the addition of $2 \mu \mathrm{l}$ DNA Gel stain. The gel was then observed in gel documentation system.

\section{Growth curve determination}

For determination of turbidity of bacterial culture method was used. Growth curve of Mccp was also determined through viable count method as described previously (Benson, 2002).

\section{Antimicrobial susceptibility test}

An antimicrobial activity of chemotherapeutics against Mccp local strain was accomplished through broth microdilution assay. Four different commercially available quinolones including Moxifloxacin, Levofloxacin, Ciprofloxacin, and Enrofloxacin were employed to sort out its effectiveness against local isolates of Mccp. The broth 
microdilution assay was carried out as described by Shah et al. (2017) and Hannan (2000). Briefly, MIC (minimum inhibitory concentration) of four above mentioned antibiotics were accomplished in sterile 96 well plate. Fresh $100 \mu 1$ PPLO broth was added to wells starting from column number $2-11$. PPLO broth $(200 \mu \mathrm{l})$ was added to column 1 as a negative control, whereas $200 \mu$ l bacterial culture was added to column 12 as positive control. Then $100 \mu 1$ of each antimicrobial was added to column 2 and serially diluted two fold to column 11 as presented in Table I. Further $100 \mu 1$ of Mccp culture $\left(0.5 \times 10^{6} \mathrm{CFU} / \mathrm{ml}\right)$ were added to each well of column 2 to 11 . The plate was then incubated at $37^{\circ} \mathrm{C}$ having $5 \% \mathrm{CO}_{2}$ and was observed periodically for growth in the positive control (column 12) and results were taken after 7 days. The experiment was performed in triplicate.

Minimum mycoplasmacidal concentration (MMC) of antimicrobials

For identification of minimum mycoplasmacidal concentration the tested broth (those wells which have no mycoplasmal growth/no color change) were subcultured on PPLO agar in petri plates and incubated at $37^{\circ} \mathrm{C}$ having $5 \% \mathrm{CO}_{2}$ and examine for Mccp colonies (Ahmad et al., 2015).

\section{Statistical analysis}

The data was analyzed by SPSS 20.0 software for a statistical significance. One-way ANOVA was carried out for checking statistical difference among various antibacterial agents used in broth microdilution assay Steel et al. (1997). LSD (least significant difference) test was carried out to split the means which were significantly different at $\mathrm{P} \leq 0.05$.

\section{RESULT}

Molecular confirmation of Mycoplasma capricolum subsp. capripneumonaie local strain by $P C R$

The PCR confirmed that the strain used in this study was belonged to Mccp species by using primers targeting 16s-rRNA gene. On gel electrophoresis of the PCR product, an amplicon size of $316 \mathrm{bp}$ was obtained that confirmed the Mccp local strain.
Growth curve of Mycoplasma capricolum subsp. capripneumoniae

The growth curve analyses of Mccp local strain confirmed that Mccp is a fastidious and slow growing specie of mycoplasma. The different phases of Mccp growth were as follow; lag phase ranges from " 0 " $\mathrm{h}$ to " 38 " h, Log/logarithmic or exponential phase ranges from " $38^{\text {th }}$ to " $72^{\text {nd }} \mathrm{h}$, the stationary phase " $72^{\text {nd }}$ to " $104^{\text {th }} \mathrm{h}$ and death/decline phase started from " $104^{\text {th }}$ h onward.

Minimum inhibition concentrations (MIC) of antimicrobial agents using broth microdilution method

For the detection of antimicrobials MIC, microtiter plate (96 well) was used and the antimicrobials were two-fold diluted as presented in Table I from lift to right, each antimicrobial agent was replicated in duplicate, and MIC was analyzed for each antimicrobial separately. The antimicrobial susceptibility assay determined that MIC value of Moxifloxacin was $2.5 \mu \mathrm{g} / \mathrm{ml}$ against Mccp, while Levofloxacin exhibited MIC value of $0.976 \mu \mathrm{g} / \mathrm{ml}$. Moreover, MIC value of ciprofloxacin was $0.3906 \mu \mathrm{g} /$ $\mathrm{ml}$, whereas Enrofloxacin had shown MIC value of 3.125 $\mu \mathrm{g} / \mathrm{ml}$ against Mccp culture (Fig. 1). Based on the assay most effective chemotherapeutic with lowest MIC value of $0.3906 \mu \mathrm{g} / \mathrm{ml}$ against Mccp was ciprofloxacin which

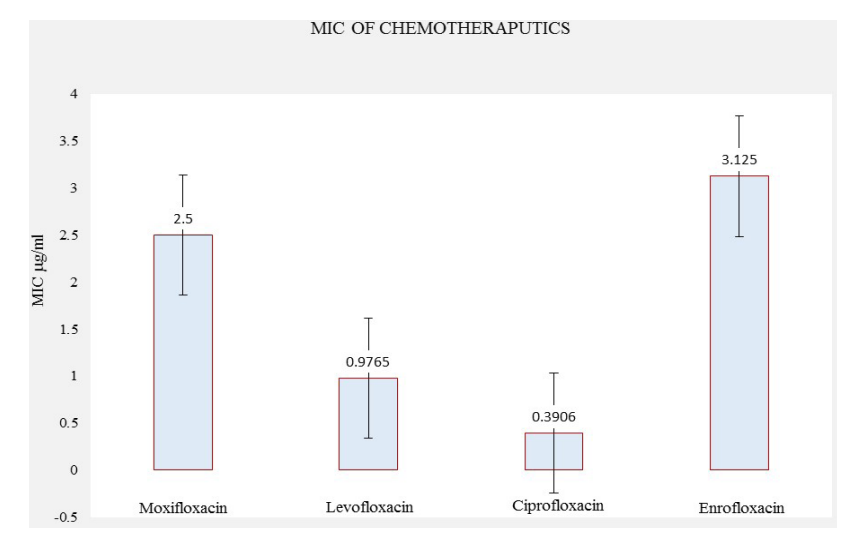

Fig. 1. MICs values of antimicrobial agents against the local isolates of Mccp. Different letters showed significant difference at $\alpha=0.05$.

Table I.- Two-fold serial dilution of chemotherapeutics used for in-vitro susceptibility trial.

\begin{tabular}{|c|c|c|c|c|c|c|c|c|c|c|c|}
\hline \multirow{2}{*}{$\begin{array}{l}\text { S No. } \\
1\end{array}$} & \multirow{2}{*}{$\begin{array}{l}\text { Antimicrobial agent } \\
\text { Moxifloxacin }\end{array}$} & \multicolumn{10}{|c|}{ Dilutions $(\mu \mathrm{g} / \mathrm{ml})$} \\
\hline & & 80 & 40 & 20 & 10 & 5 & 2.5 & 1.25 & 0.62 & 0.31 & 0.15 \\
\hline 2 & Levofloxacin & 250 & 125 & 62.5 & 31.25 & 15.62 & 7.81 & 3.90 & 1.96 & 0.97 & 0.48 \\
\hline 3 & Ciprofloxacin & 50 & 25 & 12.5 & 6.25 & 3.12 & 1.56 & 0.78 & 0.39 & 0.19 & 0.09 \\
\hline 4 & Enrofloxacin & 50 & 25 & 12.5 & 6.25 & 3.12 & 1.56 & 0.78 & 0.39 & 0.19 & 0.09 \\
\hline
\end{tabular}


showed color change from red to yellow in wells number "C10 and D10". Additionally, Ciprofloxacin also having a lowest mycoplasmacidal value of $0.7812 \mu \mathrm{g} / \mathrm{ml}$ among the tested quinolones.

Minimum mycoplasmacidal concentration (MMC) of antimicrobial by culturing tested broth

The analyses of MMC identified ciprofloxacin as most effective antibiotic with lowest MMC value of $0.781 \mu \mathrm{g} / \mathrm{ml}$ against Mccp. The MMC of Levofloxacin, Moxifloxacin, and Enrofloxacin against Mccp were 0.976 $\mu \mathrm{g} / \mathrm{ml}, 2.5 \mu \mathrm{g} / \mathrm{ml}$ and $6.25 \mu \mathrm{g} / \mathrm{ml}$, respectively.

\section{DISCUSSION}

Bacterial respiratory diseases are common in animals, especially in goats and are responsible for heavy economic loses in terms of morbidity and mortality. In bacterial disease, the mycoplasmal infections poses high risks to caprine population causing huge mortality and downgrade animal production (Onger et al., 2011; Sharif and Ghulam, 2009; Nicholas, 2002). Mycoplasma genus comprises many disease-causing species which have a propensity for respiratory organs and leading to harsh respiratory syndrome in small ruminants (Manso-Silvan et al., 2007). The respiratory disease of small ruminants is commonly treated with commercially available antibiotics having a various level of results (Laura et al., 2006). The results of most therapeutic agents are mainly based on timely reaction to disease and choosing of more suitable antibiotics with correct doses and length of treatment time. The presence of some lipoproteins on the surface of mycoplasma makes it difficult to treat because of their rapidly changing antigenic structure (Bradbury, 2005; Behrens et al., 1994). Making of biofilm is additional major escape/resistance mechanism adopted/developed by mycoplasmas against various antibiotic agents (McAuliffe et al., 2006). Commonly using antimicrobials against mycoplasma disease are oxytetracyclines, Enrofloxacin and tylosin having a distant level of results (Laura et al., 2006). Antibiotic resistance is an emerging problem of the $21^{\text {st }}$ century and holds a serious threat to public health all over the world. At the time of discovery of these chemotherapeutic agents mostly disease-causing antigen was susceptible. However, careless use of antimicrobials leaded to mutation in microorganisms, which ultimately reduced the effectiveness of broad-spectrum antimicrobials (Gautier et al., 2002; Bradbury et al., 1994). The first line of treatment recommended by US, Australia, and Europe against mycoplasma infections was macrolide. However, the efficacy of macrolides has rapidly been declined from $85 \%$ to $67 \%$ during last decade and a greater level of resistance has been found in Asia-pacific region (Shah et al., 2017; Lau et al., 2015). The second line of treatment for mycoplasma recommended by US Centers for Disease and Prevention is quinolone (Wohlkonig et al., 2010). However, there are increasing reports of resistance to quinolone in mycoplasmal infection in animals usually caused by species like M. agalactiae and M. bovis (Sulyok et al., 2014; Paterna et al., 2013; Mustafa et al., 2013). Keeping in view the above reports current research was directed to determine the different phases of Mccp growth and to detect the in-vitro effectiveness of commercially available quinolones against Mccp Pakistan strain through broth microdilution assay to find out its minimum inhibitory concentration (MIC) and minimum mycoplasmacidal concentration (MMC).

The results of current study re-confirmed that Mccp Pakistan strain is a fastidious and slow growing species of mycoplasma, till date there is no such information available on the growth curve or growth phases of Mccp Pakistan strain. Growth curve determination of Mccp Pakistan strain is helpful in numerous experiments like estimation of in vitro and in vivo growth, the expression profile of pathogen, identification of antigenic and virulence-related factors of the pathogen during different phases of growth, vaccine preparation, and evaluation of the performance of various antimicrobials. The current study also found that Mccp Pakistan strain is susceptible to all four tested members (Moxifloxacin, Levofloxacin, ciprofloxacin, Enrofloxacin) of quinolones having a different level of MIC and MMC. The investigation of current study unmasks that ciprofloxacin was a potent antimicrobial agent having lowest MICs value of $0.3906 \mu \mathrm{g} / \mathrm{ml}$ against Mccp Pakistan strain. These outcomes are in concurrence with reports about ciprofloxacin that it is the most potent antimicrobial among the tested antibacterial against the infections caused by mycoplasmas (Mustafa et al., 2013; Antunes et al., 2007). Such conclusions were also demonstrated by a researcher that ciprofloxacin is a broad spectrum antibacterial and have range activity against mycoplasma species (Kenny et al., 2001). An additional study unveiled ciprofloxacin as an efficient antimicrobial against mycoplasmal isolates having lowest MIC value of $0.06-0.5 \mu \mathrm{g} / \mathrm{ml}$ (Antunes et al., 2015). Similarly our results were further supported by the findings reported elsewhere (Antunes et al., 2007). The report indicated an in-vitro susceptibility of field isolates of Mycoplasma agalactiae and found that ciprofloxacin has the lowest MIC value of 0.125-0.5 $\mu \mathrm{g} / \mathrm{ml}$ against Mycoplasma agalactiae. Such results of ciprofloxacin with lowest MIC value of 0.05 to 1 $\mu \mathrm{g} / \mathrm{ml}$ against Mycoplasma mycoides subsp. capri was also reported previously (Paterna et al., 2016). Correspondent judgment was also described for various antimicrobials 
against Mycoplasma hominis, Mycoplasma pneumoniae and Ureaplasma urealyticum and dug out that ciprofloxacin is a potent antibiotic drug with low MIC value ranging from $0.25-1.0 \mu \mathrm{g} / \mathrm{ml}$ (Kenny and Cartwright, 2001). Being a broad spectrum antibacterial activity and due to nonavailability of ciprofloxacin for veterinary practice, it poses a strong mycoplasmacidal action against Mccp as reported through the MIC and MMC values in the current study. The results of our research reveal that broth microdilution assay is a better approach for assessing antibacterial activity. The above-mentioned assay has been flourishingly used for in vitro evaluation of chemotherapeutic agents by numerous researchers (Roberts, 1992; Taylor et al., 1997; Hannan, 2000; Mustafa et al., 2013; Jin et al., 2014).

The absence of standard CLSI-accredited MIC determining methodology for assessing of veterinary mycoplasma MIC, leading to hardness with explaining the effect of in vitro antibacterial activities. The road map approved by CLSI for other pathogen is regularly used to judge the significance of efficacy with in vitro mycoplasma sensitivity testing (Ayling et al., 2000; Francoz et al., 2005; Gerchman et al., 2009; Nicholas et al., 2003). This shows that Mccp maybe classify as sensitive to an antibacterial if it meets the sensitive category of CLSI-accredited standard for other veterinary pathogens. Therefore Levofloxacin, Moxifloxacin, and Enrofloxacin fall in susceptible criteria for respiratory pathogen at less than or equal to $2-4 \mu \mathrm{g} / \mathrm{ml}$, $1-4 \mu \mathrm{g} / \mathrm{ml}, 1-4 \mu \mathrm{g} / \mathrm{ml}$, respectively.

\section{CONCLUSIONS}

It is concluded that the Mccp Pakistan strain is susceptible to quinolone group of antibiotics. Amongst the tested quinolone, Ciprofloxacin was found the most efficacious antibiotic with lowest MIC value $(0.390 \mu \mathrm{g} / \mathrm{ml})$ and lowest MMC value $(0.781 \mu \mathrm{g} / \mathrm{ml})$, while enrofloxacin against Mccp was found showing highest MIC value of $3.125 \mu \mathrm{g} / \mathrm{ml}$ and MMC value of $6.25 \mu \mathrm{g} / \mathrm{ml}$. The broth microdilution assay is a suitable method for determination of MIC of antimicrobials.

\section{ACKNOWLEDGEMENT}

The research work was supported by the research project funded under the Pak-US Science and Technology Cooperation Program, Phase 7, 2017. This program is supported and implemented by the National Academy of Sciences (NAS) in the U.S. and by the Higher Education Commission (HEC) in Pakistan.

\section{Statement of conflict of interest}

The authors declare no conflict of interest.

\section{REFERENCES}

Antunes, N.T., Tavío, M.M., Assunção, P., Rosales, R.S., Aquili, V. and Poveda, J.B., 2007. In vitro susceptibilities of field isolates of Mycoplasma mycoides subsp. mycoides large colony type to 15 antimicrobials. Vet. Microbiol., 119: 72-75. https:// doi.org/10.1016/j.vetmic.2006.08.013

Antunes, N.T., Assunção, P., Poveda, J.B. and Tavío, M.M., 2015. Mechanisms involved in quinolone resistance in Mycoplasma mycoides subsp. Capri. Vet. J., 204: 327-332. https://doi.org/10.1016/j. tvj1.2015.04.018

Ayling, R., Baker, S. and Peek, M., 2000. Comparison of in vitro activity of danofloxacin, florfenicol, oxytetracycline, spectinomycin, and tilmicosin against field isolates of Mycoplasma bovis. Vet. Rec., 146: 745-747. https://doi.org/10.1136/ vr.146.26.745

Ahmad, I., Hao, H., Huang, L., Sanders, P., Wang, X., Chen, D., Tao, Y., Xie, S., Xiuhua, K., Li, J., Dan, W. and Yuan, Z., 2015. Integration of PK/ PD for dose optimization of Cefquinome against Staphylococcus aureus causing septicemia in cattle. Front. Microbiol., 6: 588. https://doi.org/10.3389/ fmicb.2015.00588

Behrens, A., Heller, M., Kirchhoff, H., Yogev, D. and Rosengarten, R., 1994. A family of phaseand size-variant membrane surface lipoprotein antigens (Vsps) of Mycoplasma bovis. Infect. Immun., 62: 5075-5084. https://doi.org/10.1128/ IAI.62.11.5075-5084.1994

Benson, H.J., 2002. Microbiological applications, $8^{\text {th }}$ edition. New York, pp. 926.

Bradbury, J.M., 2005. Poultry Mycoplasmas: Sophisticated pathogens in simple guise. Br. Poult. Sci., 46: 125-136. https://doi. org/10.1080/00071660500066282

Bradbury, J.M., Yavari, C.A. and Giles, C.J., 1994. Invitro evaluation of various antimicrobial against Mycoplasma gallisepticum and Mycoplasma synoviae by the broth micro method and comparison with a commercially prepared system. Avian Pathol., 23: 105-115. https://doi. org/10.1080/03079459408418978

Canton, R., Horcajada, J.P., Oliver, A., Garbajosa, P.R. and Vila, J., 2013. Inappropriate use of antibiotics in hospitals: The complex relationship between antibiotic use and antimicrobial resistance. Enfermedades Infecciosas Microbiol. Clín., 31: 3-11. https://doi.org/10.1016/S0213005X(13)70126-5 
Clothier, K.A., Kinyon, J.M. and Griffith, R.W., 2012. Antimicrobial susceptibility patterns and sensitivity to tulathromycin in goat respiratory bacterial isolates. Vet Microbiol., 156: 178-182. https://doi. org/10.1016/j.vetmic.2011.10.025

Francoz, D., Fortin, M., Fecteau, G. and Messier, S., 2005. Determination of Mycoplasma bovis susceptibilities against six antimicrobial agents using the E test method. Vet. Microbiol., 105: 5764. https://doi.org/10.1016/j.vetmic.2004.10.006

Gautier-Bouchardon, A.V., Reinhardt, A.K., Kobisch, M. and Kempf, I., 2002. In-vitro development of resistance to Enrofloxacin, erythromycin, Tylosin, tiamulin and Oxytetracycline in Mycoplasma gallisepticum, Mycoplasma iowae and Mycoplasma synoviae. Vet. Microbiol., 88: 47-58. https://doi. org/10.1016/S0378-1135(02)00087-1

Gerchman I., Levisohn S., Mikula, I. and Lysnyansky, 2009. In vitro antimicrobial susceptibility of Mycoplasma bovis isolated in Israel from local and imported cattle. Vet. Microbiol., 137: 268-275. https://doi.org/10.1016/j.vetmic.2009.01.028

Gomez-Martin, A., Amores, J. and Paterna, A., 2013. Resistance mechanisms against quinolones in Mycoplasma capricolum subsp. capricolum. Vet. J., 211: 3-7.

GoP, 2016-17. Economic survey of Pakistan, Chapter-2. Finance Division, Economic Advisors Wing, Ministry of Finance, Govt. of Pak, Islamabad, pp. 20-40.

Hannan, P., 2000. Guidelines and recommendations for antimicrobial minimum inhibitory concentration (MIC) testing against veterinary Mycoplasma species. Vet. Res., 31: 373-395. https://doi. org/10.1051/vetres:2000100

Jin, L.Y., Yyoung, M., Kyu, K.B., Man, K.J. and Lee, W.K., 2014. In-vitro antimicrobial susceptibility of Mycoplasma hyorhinis field isolates collected from swine lungs specimen in Korea. J. Swine Hlth. Prod., 22: 193-196.

Kenny, G.E. and Cartwright, F.D., 2001. Susceptibilities of Mycoplasma hominis, M. pneumoniae, and Ureaplasma urealyticum to GAR-936, dalfopristin, dirithromycin, evernimicin, gatifloxacin, linezolid, moxifloxacin, quinupristin-dalfopristin, and telithromycin compared to their susceptibilities to reference macrolides, tetracyclines, and quinolones. Antimicrob. Agents Chemother., 45: 2604-2608. https://doi.org/10.1128/AAC.45.9.2604-2608.2001

Kenny, G.E. and Cartwright, F.D., 2001. Susceptibilities of Mycoplasma hominis, Mycoplasma pneumoniae, and Ureaplasma urealyticum to a new quinolone,
OPC 17116. Antimicrob. Agents Chemother., 37: 1726-1727.https://doi.org/10.1128/AAC.37.8.1726 Lau, A., Bradshaw C.S., Lewis D., Fairley, C.K., Chen, M.Y. and Kong, F.Y., 2015. The efficacy of azithromycin for the treatment of genital Mycoplasma genitalium: A systematic review and meta-analysis. Clin. Infect. Dis., 61: 1389-1399. https://doi.org/10.1093/cid/civ644

Laura, H., Jose, L., Marcel, S.J., Lourdes, O., Jorge, A. and Katherine, H., 2006. Mycoplasma mycoides subsp. capri associated with goat respiratory disease and high flock mortality. Can. Vet. J., 47: 366-369.

Manso-Silvan, L., Perrier, X. and Thiaucourt, F., 2007. Phylogeny of the Mycoplasma mycoides cluster based on analysis of five conserved proteincoding sequences and possible implications for the taxonomy of the group. Int. J. Syst. Evol. Microbiol., 57: 2247-2258. https://doi.org/10.1099/ ijs.0.64918-0

Mathew, A.G., Cissell, R. and Liamthong, S., 2007. Antibiotic resistance in bacteria associated with food animals: A United States perspective of livestock production. Fd. Borne Pathog. Dis., 4: 115-133. https://doi.org/10.1089/fpd.2006.0066

McAuliffe, L., Ellis, R.J., Miles, K., Ayling, R.D. and Nicholas, R., 2006. Biofilm formation by Mycoplasma species and its role in environmental persistence and survival. Microbiology, 152: 913922. https://doi.org/10.1099/mic.0.28604-0

Mustafa, R., Qi, J., Ba, X., Chen, Y., Hu, C., Liu, X. and Guo, A., 2013. In vitro quinolones susceptibility analysis of Chinese Mycoplasma bovis isolates and their phylogenetic scenarios based upon QRDRs of DNA topoisomerases revealing a unique transition in ParC. Pak. Vet. J., 33: 364-369.

Nicholas, R., McAuliffe, L. and Ayling, R.D., 2003. Recent developments in the diagnosis of Mycoplasma diseases in ruminants. V. Congresso Nationale S. I. Di. L. V. Pisa, pp. 20-21.

Nicholas, R.A.J., 2002. Improvements in the diagnosis and control of diseases of small ruminants caused by Mycoplasmas. Small Rum. Res., 45: 145-149. https://doi.org/10.1016/S0921-4488(02)00095-0

OIE, 2008. Contagious caprine pleuropneumonia. Manual of standards for diagnostic tests and vaccines. Office of International Epizootics, Paris, France, pp. 1000-1012.

OIE, 2013. Contagious caprine pleuropneumonia. OIE terrestrial manual, Chapter, 2.7.6. Office of International Epizootics, Paris, France, pp. 10001012. 
Ongor, H., Kalin, R. and Acik, M.N., 2011. Detection of Mycoplasma ovipneumoniae from goats with nasal discharge by culture and polymerase chain reaction. Pak. Vet. J., 31: 244-248.

Paterna, A., Sanchez, A., Gomez-Martin, A., Corrales, J.C., de la Fe, C., Contreras, A. and Mores, J., 2013. In vitro antimicrobial susceptibility of Mycoplasma agalactiae strains isolated from dairy goats. $J$. Dairy Sci., 96: 7073-7076. https://doi.org/10.3168/ jds.2012-6492

Paterna, A., Tatay-Dualde, J., Amores, J., Prats-van der Ham, M., Sánchez, A., de la Fe, C. and GómezMartín, A., 2016. In vitro assessment of the antimicrobial susceptibility of caprine isolates of Mycoplasma mycoides subsp. capri. Vet. J., 214: 96-101. https://doi.org/10.1016/j.tvj1.2016.05.010

Rehman, A., Jingdong, L., Chandio, A.A. and Hussain, I., 2017. Livestock production and population census in Pakistan: Determining their relationship with agricultural GDP using econometric analysis. Inform. Process. Agric., 4: 168-177. https://doi. org/10.1016/j.inpa.2017.03.002

Reynolds, J. and Farinha, M., 2005. Colony counting. Richland College.

Roberts, M.C., 1992. Antibiotics resistance in Mycoplasmas: Molecular biology and pathogenesis (eds. J. Maniloff, R.N. McElhaney, L.R. Finch. and J.B. Baseman). American Society for Microbiology, Washington, DC, pp. 513-523.

Scott, L.C. and Menzies, P.I., 2011. Antimicrobial resistance and small ruminant veterinary practice. Vet. Clin. N. Am. Fd. Anim. Pract., 1: 23-32. https:// doi.org/10.1016/j.cvfa.2010.10.015

Shah, M.K., Saddique, U., Ahmad, S., Iqbal, A., Ali, A., Shahzad, W., Khan, S. and Rehman, H.U., 2017. Molecular characterization of local isolates of Mycoplasmas capricolum sub species capripneumoniae in goats (Capra hircus) of Khyber Pakhtunkhwa, Pakistan. Pakistan Vet. J., 37: 90-94.

Sharif, A. and Ghulam, M., 2009. Mastitis control in dairy animals. Pak. Vet. J., 29: 145-148.

Steel, R.G.D., Torrie, J.H. and Dieky, D.A., 1997. Principles and procedures of statistics, $3^{\text {rd }}$ ed. McGraw-Hill Book Co. Inc., New York.

Sulyok, K.M., Kreizinger, Z., Fekete, L., Hrivnak, V., Magyar, T., Janosi, S., Schweitzer, N., Turcsanyi, I., Makrai, L. and Erdelyi, K., 2014. Antibiotic susceptibility profiles of Mycoplasma bovis strains isolated from cattle in Hungary, Central Europe. BMC Vet. Res., 10: 256. https://doi.org/10.1186/ s12917-014-0256-X

Swai, E.S., Kaaya, J.E. and Noah, E.Y., 2013. Antibody response to Mycoplasma capricolum subsp. capripneumoniae bacterium in small holder dairy goats in Tanzania. Trop. Anim. Hlth. Prod., 45: 1603-1608. https://doi.org/10.1007/s11250-0130405-4

Tabatabaei-Qomi, R., Sheykh-Hasan, M., Fazaely, H., Kalhor, N. and Ghiasi, M., 2014. Development of a PCR assay to detect mycoplasma contamination in cord blood hematopoietic stem cells. Iranian J. Microbiol., 6: 281.

Taylor-Robinson, D. and Bebear, C., 1997. Antibiotic susceptibilities of Mycoplasmas and treatment of Mycoplasmal infections. J. Antimicrob. Chemother., 40: 622-630. https://doi.org/10.1093/ jac/40.5.622

Wohlkonig A., Chan, P.F., Fosberry, A.P., Homes, P., Huang, J. and Kranz, M., 2010. Structural basis of quinolone inhibition of type IIA topoisomerases and target-mediated resistance. Nat. Struct. mol. Biol., 17: 1152-1153. https://doi.org/10.1038/ nsmb. 1892

Woubit, S., Lorenzon, S., Peyraud, A., Manso-Silvan, L. and Thiaucourt, F., 2004. A specific PCR for the identification of Mycoplasma capricolum subsp. capripneumoniae, the causative agent of contagious caprine pleuropneumonia (CCPP). Vet. Microbiol., 104: 125-132. https://doi.org/10.1016/j. vetmic.2004.08.006 